\title{
Synchronous Distal Pancreatic Metastatic Lesion Arising from Colonic Adenocarcinoma: Case Report and Literature Review
}

Lowell Su, MD and Jessica Wernberg, MD

\begin{abstract}
Solitary metastatic pancreatic lesions comprise $0.5 \%$ to $3 \%$ of all pancreas neoplasms, most commonly arising from primary tumors of the kidney, lung, or colon. Synchronous metastatic pancreatic lesions are exceptionally rare. Only 25 cases of isolated colorectal pancreatic metastasis amenable to resection have been reported, II of those in the distal pancreas. To our knowledge we report the first case of a primary colonic malignancy with a synchronous distal pancreatic metastatic lesion treated with resection.
\end{abstract}

Keywords: Colonic neoplasm; Pancreatic neoplasm; Pancreatic metastasis, Distal pancreatectomy

Corresponding Author:

Lowell Su, MD

Department of General Surgery

Marshfield Clinic

1000 North Oak Avenue

Marshfield, WI 54449

Tel: (7I5) 387-9222

Fax: (7I5) 389-4454

Email: su.lowell@marshfieldclinic.org

Received: August 22, 2013

Revised: October 14, 2013

Accepted: October 17.2013

doi: $10.3121 / \mathrm{cmr} .2013 .1 \mid 95$
$O$ metastatic pancreatic lesions comprise $0.5 \%$ to $3 \%$ of all pancreas neoplasms. ${ }^{1}$ These tumors arise most commonly from primary tumors of the kidney, lung, or colon. ${ }^{2}$ Other primary tumors associated with metastatic pancreatic lesions include breast cancer, skin melanomas, soft tissue sarcomas, and gastrointestinal tumors. Following detection of the primary tumor, most cases of isolated metastatic pancreatic tumors present within a certain time interval defined by the primary malignancy. The median time interval for colorectal primary tumors is 24 months, while metastasis from renal cell carcinoma is 120 months. $^{3}$ Synchronous metastatic pancreatic lesions are exceptionally rare, with only three other cases previously reported, none of which involved the distal pancreas. $^{2}$

Studies have shown survival benefit for those patients undergoing surgical resection for hepatic, lung, and brain metastasis from primary colorectal malignancies. However, due to the rarity of metastatic pancreatic lesions, no survival benefit has yet been shown for metastatic solitary pancreatic lesion resections. ${ }^{4}$ Historically, operative risk for all metastatic pancreatic lesion resection was too high to justify therapeutic metastasectomy. Recently, however, a push toward aggressive metastasectomy of isolated pancreatic masses has been advocated. ${ }^{5}$ To date, only 25 cases of colorectal solitary metastasis to the pancreas amenable to resection have been reported. Eleven of those cases occurred in the distal pancreas, and only three cases were synchronous metastatic lesions. To our knowledge we report the first case of a primary colon malignancy with an isolated synchronous distal pancreatic metastatic lesion simultaneously treated with surgical resection. 


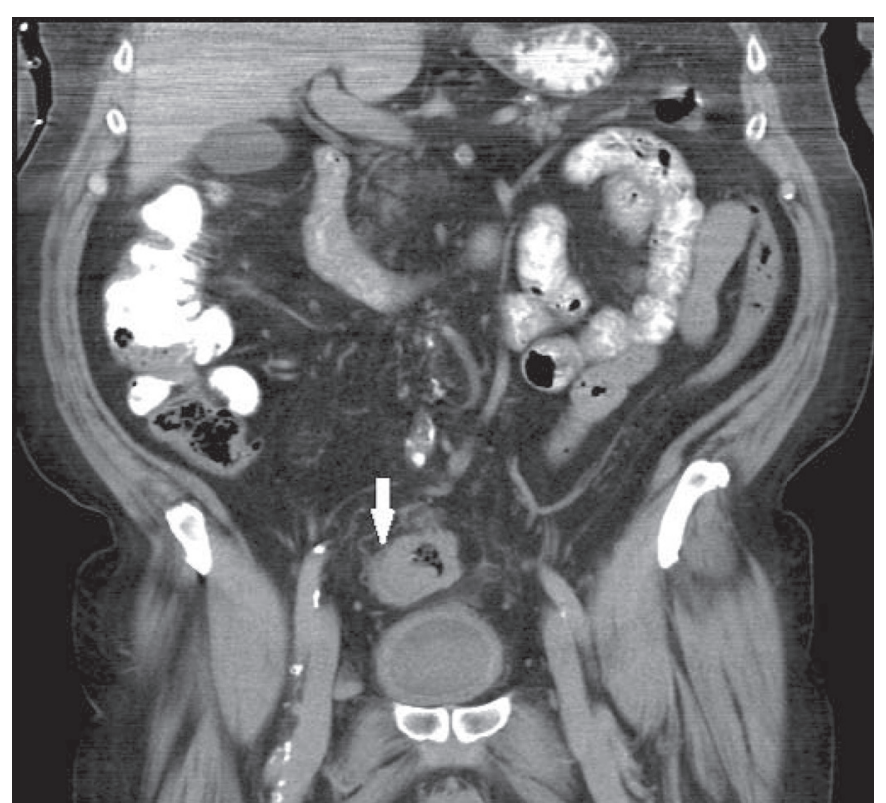

Figure 1. Computed tomography (CT), axial view, performed with oral and intravenous contrast shows an obstructing sigmoid mass with surrounding inflammatory changes and enlarged local lymph nodes.

\section{Case Report}

A male patient, aged 73 years, was referred to our clinic with epigastric pain exacerbated by meals and bright red blood per rectum with narrow caliber dark stools over several months. His history was significant for recent coronary artery bypass grafting $(\mathrm{CABG})$ one year prior to presentation and postoperative atrial fibrillation requiring cardioversion and anticoagulation. There was no family history of cancer. He had a remote smoking history of 40 pack years and had never undergone screening colonoscopy.

On physical examination the patient's abdomen exhibited mild epigastric tenderness with no palpable masses, but melenic stools on rectal examination. An abdominal ultrasound revealed no focal pancreatic mass, and hepatobiliary iminodiacetic acid scan (HIDA) showed a normal gallbladder ejection fraction with negative biliary pathology. Esophagogastroduodenoscopy (EGD) was unremarkable. Colonoscopy revealed a nearly occlusive annular sigmoid mass $20 \mathrm{~cm}$ from the anus and seven diminutive polyps, scattered throughout the entire colon. Biopsies of the mass lesion were positive for moderately differentiated invasive colon adenocarcinoma with ulceration, while the polyps removed with hot biopsy polypectomy were not recovered for pathology. Clinical staging was performed utilizing computed tomography (CT) of the abdomen and confirmed an annular sigmoid colonic mass with enlarged local lymph nodes (figure 1). A solid lesion measuring $2.3 \times 2.5 \mathrm{~cm}$ was seen in the pancreatic tail with no obvious lymph node involvement (figure 2). Subsequent positron emission tomography (PET) scan confirmed CT findings of cancer in the sigmoid colon with an additional lesion in the pancreatic tail (figures 3 and 4). The patient was diagnosed with colon cancer with a likely second primary neoplasm of the pancreas and was felt to be an operative candidate for concomitant resection.

Intraoperatively, the abdomen was entered in an open fashion. The sigmoid mass was identified with clear involvement of the mesentery and sigmoid colectomy with primary anastomosis was performed. The pancreas was accessed via the lesser sac, and the mass was identified along the distal inferior pancreatic border. There was no other grossly visible retroperitoneal involvement. The mass was resected and the spleen was sacrificed. The pathology identified local invasive sigmoid adenocarcinoma with 3 of 14 pericolonic lymph nodes positive for metastasis. There were both local vascular and perineural invasion in the colon and pancreas. Twentythree peripancreatic lymph nodes were negative for metastasis. The pancreatic mass tested positive for CK7, CK20, and CDX2 expression by immunohistology, confirming the diagnosis of a metastatic colon adenocarcinoma lesion to the pancreas. Tumor staging was classified as stage IV (pT4aN1bM1a).

Postoperatively, the patient's hospital course was complicated by bradycardia and hypotension requiring prolonged intensive care unit (ICU) care. He recovered and was discharged in sinus rhythm without anticoagulation and was seen 3 months later in the surgical clinic with no complications. He is scheduled to receive adjuvant chemotherapy for his metastatic colon cancer.

\section{Discussion}

Whereas colon cancer metastasis to the pancreas is rare, synchronous pancreatic spread is exceptionally rare. Common metastatic sites for colon adenocarcinoma include liver, lung, peritoneum, bone, brain, and less commonly, adrenals, testicles, and skin. The pancreas is also a recognized site for

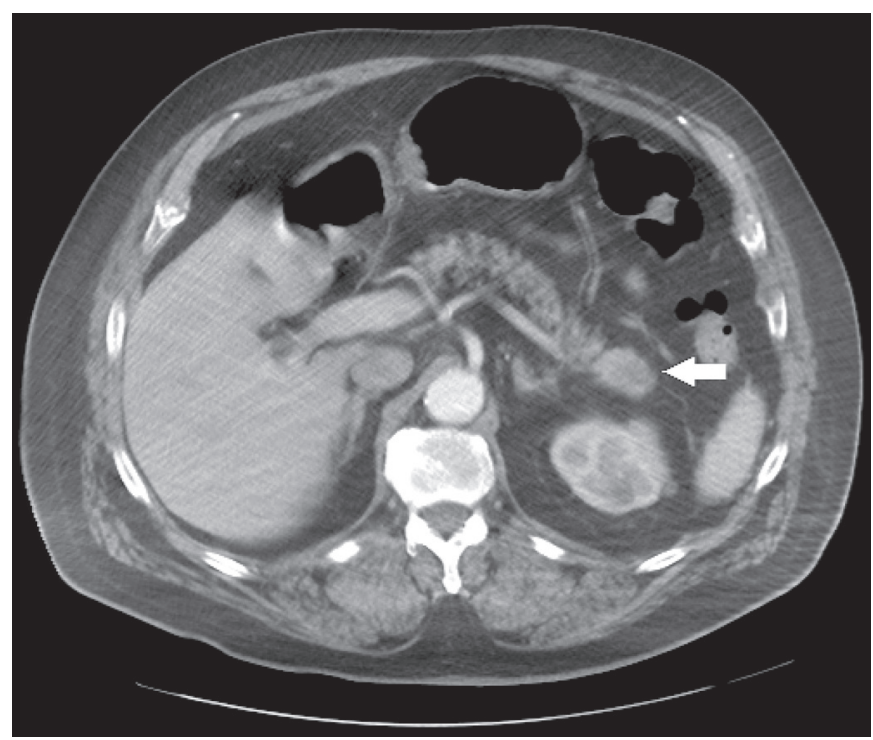

Figure 2. Computed tomography (CT), axial view, performed with oral and intravenous contrast shows a distal pancreatic lesion measuring $2.3 \times 2.5 \mathrm{~cm}$ with no obvious lymph node involvement. 


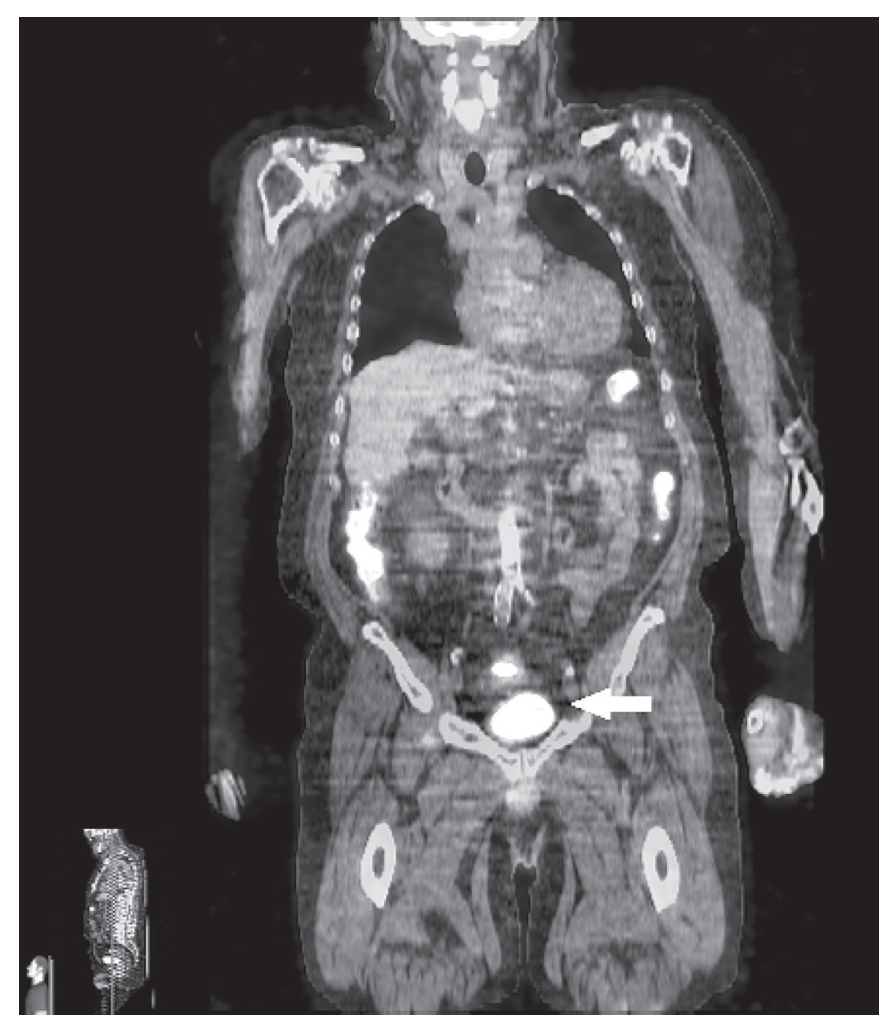

Figure 3. Positron emission tomography (PET) showing clear increased metabolic uptake of fludeoxyglucose (FDG) in the same site as the sigmoid mass seen previously on computed tomography (CT).

colon cancer metastasis. The overall incidence of pancreatic metastatic neoplasms in autopsy series was found to be $1.6 \%$ to $11 \%$, and $3.9 \%$ in all pancreas resections. ${ }^{6}$ Roland et $\mathrm{al}^{7}$ reviewed 1357 patients with synchronous discovery of pancreatic tumors and described only 27 patients $(2 \%)$ with pancreatic metastasis. Only 4 patients $(0.3 \%)$ underwent surgical metastasectomy. ${ }^{7}$ A review of 13 reports (11 single patient reports and 2 with 2 patients each) found only 24 patients who underwent metastasectomy for metastatic colon cancer. Among these, solitary pancreatic metastasis was present in 17/24 patients, and 7/24 patients had other metastases that required at least one additional associated surgical procedure. ${ }^{8-19}$ Sperti et $\mathrm{al}^{20}$ reported that review of their data on pancreatic metastatic lesions found only 18/546 of these lesions (3.2\%) had indications for metastasectomy, and half of these had colon cancer as the primary malignancy. To date, only three cases of synchronous colorectal metastasis to the pancreas have been reported, two occurring in the head of the pancreas and one occurring in the body. ${ }^{2}$

A firm understanding of distant metastasis distribution patterns from primary colorectal neoplasms is important for clinical cancer diagnosis and treatment. There are two prevailing hypotheses that explain the metastatic patterns of colonic adenocarcinomas: the mechanical/hemodynamic theory and the seed-and-soil theory. ${ }^{21}$ The mechanical theory has its basis in anatomical delivery via the venous and lymphatic drainage systems of the body. The theory explains the spread of colonic tumors via emboli that drain either into the portal system and metastasize into the liver or by systemic routes into the lung. McDaniel et $\mathrm{al}^{22}$ found that pancreatic metastasis from colonic adenocarcinomas tended to be from right-sided colonic tumors. This observation is consistent with the mechanical spread theory, which postulates that cecal tumors may spread to the pancreas directly via ileocolic and superior mesenteric vessels. The seed-and-soil theory was coined by Stephen Paget and is based on metastasizing tumor cells finding a tissue bed that is congenial for its deposit and growth. ${ }^{23}$ This theory prompted the current molecular genetic microassay studies that examine gene expressions to help explain cancer cell predilection for certain metastatic sites and specific molecular receptors on distant cells that allow tumor emboli to attach. Though not all encompassing, the seed-and-soil theory offers plausible insights regarding unique metastatic sites not found along normal anatomic drainage routes. Given the paucity of reports of colonic adenocarcinomas spreading to the pancreas, the mechanism of this particular metastasis is not fully understood. While each theory may offer plausible mechanisms explaining our patient's synchronous metastatic distal pancreatic tumor, ongoing research of metastatic patterns of adenocarcinoma at a molecular level ultimately will provide more definitive insights.

Clinical diagnosis of solitary metastatic pancreatic tumors remains challenging, as these tumors comprise only $0.5 \%$ to $3 \%$ of all pancreatic neoplasms. Metastatic pancreatic tumors

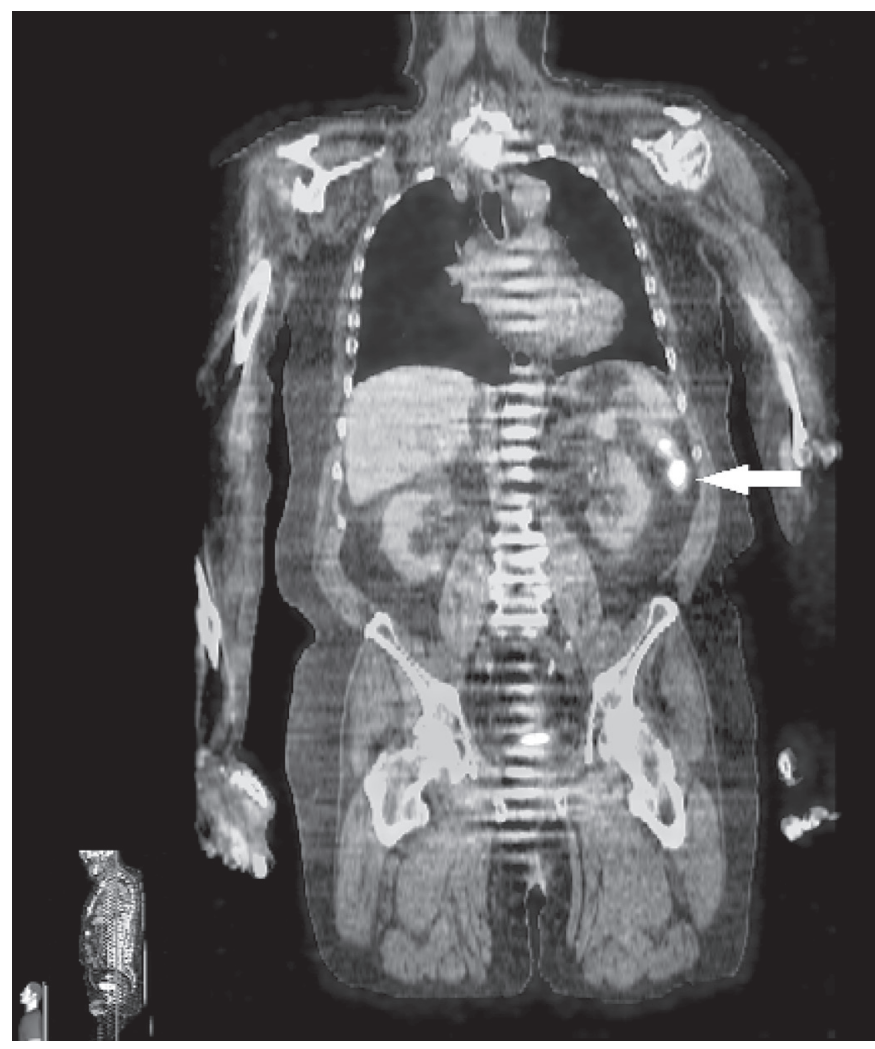

Figure 4. Positron emission tomography (PET) showing an additional lesion with increased fludeoxyglucose (FDG) uptake in the distal pancreas. 
are asymptomatic in $35 \%$ of all cases of renal cell carcinoma and present asymptomatically in $71 \%$ of colorectal tumors. ${ }^{3}$ In patients who present with symptoms, unique presentation associated with isolated metastatic pancreatic lesions distinguishes them from patients with primary pancreatic tumors who frequently present with abdominal pain, weight loss, and jaundice. Lee et $\mathrm{al}^{2}$ examined symptomology of all patients with an isolated metastatic pancreatic lesion arising from colorectal adenocarcinoma. The incidence of particular symptoms was: abdominal pain (20\%), weight loss (5\%), and jaundice $(30 \%)$. Interestingly, $54.5 \%$ of those patients with metastatic tumors in the head of the pancreas did not present with jaundice. ${ }^{2}$

Recommended imaging studies for both symptomatic and asymptomatic pancreatic mass include abdominal ultrasound and contrast CT. While CT can potentially distinguish between renal cell carcinoma, pancreatic metastasis, and primary pancreatic tumors, this is not true for colorectal primary lesions. ${ }^{3}$ No greater ability to distinguish between primary and metastatic pancreatic lesions has been shown with MRI than with CT or ultrasound. ${ }^{8,24}$

There has been controversy concerning whether pre-treatment diagnosis of pancreatic tumors via tissue biopsy is necessary to inform treatment decisions. Proponents advocate for accurate diagnosis for staging purposes, while opponents argue that percutaneous biopsy poses a higher risk of tumor cell dissemination. In patients with resectable pancreatic lesions in the head or tail, the risk/benefit ratio should be carefully considered. Baierlein et $\mathrm{al}^{24}$ projected that endoscopic ultrasound guided fine-needle aspiration (EUS-FNA) in patients with colorectal metastasis to the pancreas has a diagnostic accuracy of $84 \%$ to $95 \%$. They proposed the following indications for pretreatment EUS-FNA: (1) all other diagnostic measures failed; (2) pathological examination demonstrates certain markers or gene mutations that are needed for initiation of specific treatments; (3) biopsy results will influence therapy; (4) biopsy results can avoid or minimize surgery; and (5) loco-regional staging of pancreatic tumors. Given the circumstances of the present case, need for a biopsy was precluded based on the following rationale: the patient had a known sigmoid cancer requiring operation; he presented simultaneously with a distal pancreatic tumor amenable to resection based on imaging studies; and he was felt to be a candidate for concomitant resection. Preoperative diagnosis for this particular pancreatic tumor would not have changed our therapeutic approach, and EUS-FNA biopsy was not pursued; although, we are in agreement with the aforementioned indications for EUS-FNA. We would further propose that the location of the pancreatic lesion may additionally influence the decision to pursue EUS-FNA. Pancreatic head masses can be accessed via the duodenum and are theoretically without risk for seeding. Further, the tumor histology may influence whether surgical intervention or neoadjuvant chemotherapy is pursued as initial treatment.
Identification of the primary site in patients with potential metastatic adenocarcinoma or greater than two unknown tumors is paramount for prognosis and therapy. Immunohistochemistry has helped provide accurate diagnosis of unknown tumors using molecular markers. Markers expressed in colon adenocarcinoma include CK20 and CDX2. CK20 is expressed in most gastrointestinal tumors-including primary colonic, pancreatic, and gastric cancers - but is nonspecific. CDX2 is also found in colon adenocarcinoma, with only very low detection levels in most gastric and pancreatic tumors. ${ }^{25}$ Using these markers, we were able to accurately diagnosis our patient with a solitary metastatic pancreas tumor originating from the sigmoid colon.

The role of surgical resection for solitary metastatic pancreatic tumors is not well defined; however, recent literature supports resection. Overall, 25 solitary pancreatic metastatic lesions from colorectal malignancy have been described, of which 11 were found in the distal pancreas, and 3 were synchronous tumors. Since metastasectomies of the brain, lung, and liver have been associated with increased overall survival of patients with colorectal adenocarcinomas, metastasectomy of solitary pancreas metastasis is supported. In a recent review, Sperti et $\mathrm{al}^{20}$ cited no perioperative morbidity or mortality in surgical procedures involving solitary pancreatic metastases, with an overall median survival time for these patients of 16.5 months. Disease-free survival reported in five studies ranged from 1.5 to 43 months, and $100 \%$ of symptomatic patients had complete symptom relief until recurrence or death. ${ }^{3}$ These data suggest that surgical resection may, at a minimum, improve patient quality of life. Future studies regarding survival benefit will need to be reported.

There are no prospective or retrospective studies comparing operative resection of isolated pancreatic metastases from colorectal carcinoma with nonoperative chemotherapeutic, neoadjuvant chemotherapeutic, or palliative approaches. Adjuvant systemic chemotherapy was recommended to our patient, although specific data suggesting improved survival or quality of life is lacking. Furthermore, the significance of the synchronous nature of his metastatic disease on survival and recurrence is unclear.

The three other synchronous pancreatic tumors reported in the literature that were derived from colorectal malignancy arose in the pancreatic head in two patients and the body in one patient. One of the patients with a pancreatic head metastasis achieved 28+ months of survival following diagnosis and was alive at the time of publication; whereas the other patient succumbed 15 months following diagnosis. The patient with the pancreatic body malignancy succumbed 5 months post diagnosis. To our knowledge no synchronous pancreatic tail solitary metastasis has been reported to date. Our patient did have colonic and peripancreatic perineural and lymphovascular invasion with increased risk for recurrence and shorter survival. Whether his ongoing adjuvant therapy will provide better outcomes is unknown. 


\section{Conclusion}

We presented a unique case report of an extremely rare case of synchronous distal pancreatic metastasis from a colonic primary adenocarcinoma. Pancreatic metastases should be part of the differential diagnosis when a patient presents with a primary colorectal cancer and a pancreatic mass. When possible, surgical resection of both tumors should be offered concomitantly to optimize quality of life, though benefit to overall survival is currently unknown. Extrapolating from lung and liver metastasectomy data would suggest it is a reasonable approach in appropriate patients. Long-term follow-up is essential in monitoring for recurrence and efficacy of the chosen therapeutic approach.

\section{References}

1. Lasithiotakis K, Petrakis I, Georgiadis G, Paraskakis S, Chalkiadakis G, Chrysos E. Pancreatic resection for metastasis to the pancreas from colon and lung cancer, and osteosarcoma. JOP 2010;11:593-596.

2. Lee CW, Wu RC, Hsu JT, Yeh CN, Yeh TS, Hwang TL, Jan YY, Chen MF. Isolated pancreatic metastasis from rectal cancer: a case report and review of literature. World J Surg Oncol 2010;8:26.

3. Machado NO, Chopra PJ, Hamdani A. Pancreatic metastasis from colon carcinoma nine years after a hemicolectomy managed by distal pancreatectomy. A review of the literature regarding the role and outcome of pancreatic resection for colorectal metastasis. JOP 2010;11:377-381.

4. Turk PS, Wanebo HJ.: Results of surgical treatment of nonhepatic recurrence of colorectal carcinoma. Cancer 1993;71(12 Suppl):4267-77.

5. Sperti C, Pasquali C, et al.: Pancreatic resection for metastatic tumors to the pancreas. J Surg Oncol 2003;83(3):161-6.

6. Adsay NV, Andea A, Bastruk O, Lilinc N, Nassar H, Cheng JD. Secondary tumors of the pancreas: an analysis of a surgical and autopsy database and review of literature. Virchows Arch 2004;444:527-535.

7. Roland CF, van Heerden JA. Nonpancreatic primary tumors with metastasis to the pancreas. Surg Gynecol Obstet 1989; $168: 345-347$.

8. Inagaki H, Nakao A, Ando N, Kotake K, Imaizumi T, Okuda N, Kaneko T, Kurokawa T, Nonami T, Takagi H. A case of solitary metastatic pancreatic cancer from rectal carcinoma: a case report. Hepatogastroenterology 1998;45:2413-2417.

9. Matsubara N, Baba H, Okamoto A, Kurata M, Tsuruta K, Funata $\mathrm{N}$, Ashizawa K. Rectal cancer metastasis to the head of the pancreas treated with pancreaticoduodenectomy. J Hepatobiliary Pancreat Surg 2007;14:590-594.

10. Tutton MG, George M, Hill ME, Abulafi AM.: Solitary pancreatic metastasis from a primary colonic tumor detected by PET scan: report of a case. Dis Colon Rectum 2001;44:288-290.

11. Le Borgne J, Partensky C, Glemain P, Dupas B, de Kerviller B. Pancreaticoduodenectomy for metastatic ampullary and pancreatic tumors. Hepatogastroenterology 2000;47:540-544.

12. Nakeeb A, Lillemoe KD, Cameron JL. The role of pancreaticoduodenectomy for locally recurrent or metastatic carcinoma of the periampullary region. J Am Coll Surg 1995;180:188-192.

13. Harrison LE, Merchant N, Cohen AM, Brennan MF. Pancreaticoduodenectomy for nonperiampullary primary tumors. Am J Surg 1997;174:393-395.

14. Yoshimi F, Asato Y, Kuroki Y, Shioyama Y, Hori M, Itabashi M, Amemiya R, Koizumi S. Pancreaticoduodenectomy for locally advanced or recurrent colon cancer: report of two cases. Surg Today 1999;29:906-910.
15. Torres-Villalobos G, Podgaetz E, Anthon FJ, Remes-Troche JM, Robles-Diaz G, Nuñez CC. Single pancreatic metastasis from a previously resected carcinoma of the cecum: a case report. Curr Surg 2004;61:328-330.

16. Crippa S, Angelini C, Mussi C, Bonardi C, Romano F, Sartori P, Uggeri F, Bovo G. Surgical treatment of metastatic tumors to the pancreas: a single center experience and review of the literature. World J Surg 2006;30:1536-1542.

17. Eidt S, Jergas M, Schmidt R, Siedek M. Metastasis to the pancreas--an indication for pancreatic resection? Langenbecks Arch Surg 2007;392:539-542.

18. Shimoda M, Kubota K, Kita J, Katoh M, Iwasaki Y. Is a patient with metastatic pancreatic tumor from rectal cancer a candidate for resection? Hepatogastroenterology 2007;54:1262-1265.

19. Bachmann J, Michalski CW, Bergmann F, Büchler MW, Kleeff J, Friess H. Metastasis of rectal adenocarcinoma to the pancreas: two case reports and a review of the literature. JOP 2007;8:214-222.

20. Sperti C, Pasquali C, Berselli M, Frison L, Vicario G, Pedrazzoli S. Metastasis to the pancreas from colorectal cancer: is there a place for pancreatic resection? Dis Colon Rectum 2009;52:1154-1159.

21. Hess KR, Varadhachary GR, Taylor SH, Wei W, Raber MN, Lenzi R, Abbruzzese JL. Metastatic patterns in adenocarcinoma. Cancer 2006;106:1624-1633.

22. McDaniel KP, Charnsangavej C, DuBrow RA, Varma DG, Granfield CA, Curley SA. Pathways of nodal metastasis in carcinomas of the cecum, ascending colon, and transverse colon: CT demonstration. AJR Am J Roentgenol 1993;161:61-64.

23. Paget $\mathrm{S}$. The distribution of secondary growths in cancer of the breast. 1889. Cancer Metastasis Rev 1989;8:98-101.

24. Baierlein SA, Wistop A, Looser C, Bussmann C, von Flüe M, Peterli R. Primary pancreatic neoplasia or metastasis from colon carcinoma? Acta Gastroenterol Belg 2008;71:401-408.

25. Dennis JL, Hvidsten TR, Wit EC, Komorowski J, Bell AK, Downie I, Mooney J, Verbeke C, Bellamy C, Keith WN, Oien KA. Markers of adenocarcinoma characteristic of the site of origin: development of a diagnostic algorithm. Clin Cancer Res 2005;11:3766-3772.

\section{Author Affiliations}

Lowell Su, MD*; Jessica Wernberg, $M D^{\prime}$

*Surgical Resident, Department of General Surgery, Marshfield Clinic, Marshfield, Wisconsin, USA Assistant Program Director, Department of General Surgery, Marshfield Clinic, Marshfield, Wisconsin, USA 